Y. Kitaoka

Nagoya Math. J.

Vol. 66 (1977), 139-149

\title{
SCALAR EXTENSION OF QUADRATIC LATTICES
}

\author{
YOSHIYUKI KITAOKA
}

Let $E / F$ be a finite extension of algebraic number fields, $O_{E}, O_{F}$ the maximal orders of $E, F$ respectively. A classical theorem of Springer [6] asserts that an anisotropic quadratic space over $F$ remains anisotropic over $E$ if the degree $[E: F]$ is odd. From this follows that regular quadratic spaces $U, V$ over $F$ are isometric if they are isometric over $E$ and $[E: F]$ is odd. Earnest and Hsia treated similar problems for the spinor genera [2,3]. We are concerned with the quadratic lattices. Let $L, M$ be quadratic lattices over $O_{F}$ in regular quadratic spaces $U, V$ over $F$ respectively. Assume

(*) there is an isometry $\sigma$ from $O_{E} L$ onto $O_{E} M$, where $O_{E} L, O_{E} M$ denote the tensor products of $O_{E}$ and $L, M$ over $O_{F}$ respectively. Then our question is whether the assumption implies $\sigma(L)=M$ or not. The affirmative answer would imply that $L, M$ are already isometric over $O_{F}$.

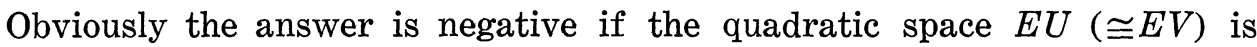
indefinite. Even if we suppose that $E U$ is definite, the answer is still negative in general. However there are many cases in which the answer is affirmative if $E U$ is definite. We give such examples in this paper.

Through this paper $Q(x), B(x, y)$ denote quadratic forms and corresponding bilinear forms $(2 B(x, y)=Q(x+y)-Q(x)-Q(y))$. Notations and terminologies will be those of O'Meara [5].

THEOREM 1. Let $m$ be a natural number $\geq 2$, and $E$ be a totally real algebraic number field with degree $m$, and assume that $L, M$ be definite quadratic lattices over the ring $\boldsymbol{Z}$ of rational integers. Then the assumption*) $(*)$ implies $\sigma(L)=M$ if $E$ does not intersect with $a$ finite set of (explicitely determined) algebraic integers which are not dependent on $L, M$, but on $m$.

THEOREM 2. Let $E$ be totally real, and $L, M$ be definite quadratic

Received May 20, 1976.

*) In Theorem 1, 2, and $3 F$ is the field $Q$ of rational numbers. 
lattices over $Z$ with $\operatorname{rank} L=\operatorname{rank} M \leq 5$. The assumption ${ }^{*}$ (*) implies $\sigma(L)=M$ if $E$ does not contain any of $\sqrt{2}, \sqrt{3}$ and $\sqrt{5}$ in case of $\operatorname{rank} L=\operatorname{rank} M=5$.

COROLlARY. Let $E, K$ be a totally real algebraic number field and an imaginary quadratic field respectively whose discriminants are relatively prime. Then an ideal of $K$ is principal if it is principal in the composite field $K E$.

THEOREM 3. Let $E$ be a totally real algebraic number field with $[E: Q] \leq 5$, and $L, M$ be definite quadratic lattices over $Z$. Then the assumption $^{*}(*)$ implies $\sigma(L)=M$.

In case that $L=M$ and $\sigma$ is associated with an orthogonal decomposition of $O_{E} L$ we have

THEOREM 4. Let $E / F$ be a Galois extension of totally real algebraic number fields. Assume that $F$ is the only field between $E$ and $F$ which is unramified over $F$. If a definite quadratic lattice $L$ over $O_{F}$ is decomposable over $O_{E}$, i.e., $O_{E} L=L_{1}^{\prime} \perp L_{2}^{\prime}$, then there is a decomposition of $L, L=L_{1} \perp L_{2}$, with $L_{i}^{\prime}=O_{F} L_{i}(i=1,2)$, in other words, a definite indecomposable quadratic lattice over $O_{F}$ remains indecomposable over $O_{E}$.

COROLLARY. Let $E$ be a totally real algebraic number field, and $L$ be a definite indecomposable quadratic lattice over $Z$. Then $O_{E} L$ is also indecomposable.

We give some other sufficient conditions to the affirmative answer of our question.

THEOREM 5. Let $F$ be totally real and $E=F(\sqrt{a})$ be a totally real quadratic extension, and let $L, M$ be definite quadratic lattices over $O_{F}$ and suppose that there is an isometry $\sigma$ from $O_{E} L$ onto $O_{E} M$. Then, one of the following conditions on $O_{E}$ :

(i) $O_{E}=O_{F}+A \sqrt{a}$, where $A$ is an ideal of $F$ such that $A^{2} a \neq O_{F}$,

(ii) $O_{E}=O_{F}+A x$, where $A$ is an ideal of $F, x^{2} \notin F$, and $N_{E / F} x$ is totally negative,

implies $\sigma(L)=M$.

THEOREM 6. Let $E / F$ be a Galois extension of totally real algebraic number fields, and assume that $F$ is the only field between $E$ and $F$ 
which is unramified over $F$. Let $L, M$ be definite quadratic lattices over $O_{F}$ and let $\sigma$ be an isometry from $O_{E} L$ onto $O_{E} M$. Then we get $\sigma(L)$ $=M$ if $\sigma(L) \subset M+2 O_{E} M$.

1.1. Let $E$ be a totally real algebraic number field with degree $m$, and $L$ be a positive definite quadratic lattice over $Z$ with $\operatorname{rank} n \leq m$.

LEMma. Let $v_{0}$ be an element in $O_{E} L$ such that $Q\left(v_{0}\right)=\min Q(v)$, where $v$ runs over non-zero elements of $O_{E} L$ with $Q(v) \in \boldsymbol{Q}$. Then $v_{0}$ is in $L$ if $E$ does not intersect with a finite set of (explicitely determined) algebraic integers which are not dependent on $L$ but $m$.

Proof. We denote by $B($,$) the bilinear form associated with L$ as indicated in the introduction. There exists a $Z$-basis $\left\{e_{i}\right\}$ of $L$ such that

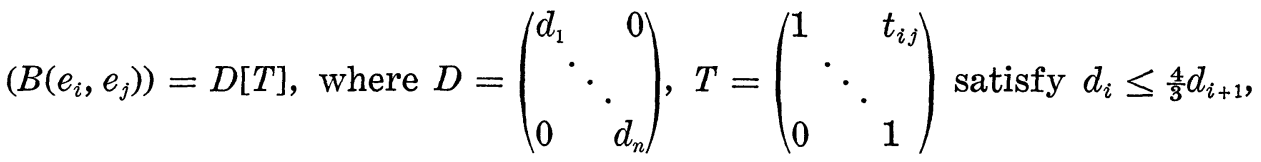
$(i=1, \cdots, n-1),\left|t_{i j}\right| \leq \frac{1}{2},(i<j),\left(p .20\right.$ in [1]). Put $v_{0}=\sum x_{i} e_{i}\left(x_{i} \in\right.$ $\left.O_{E}\right)$, and $\boldsymbol{y}=\left(\begin{array}{c}y_{1} \\ \vdots \\ y_{n}\end{array}\right)=\left(\begin{array}{ccc}1 & & t_{i j} \\ & \ddots & \\ & & 1\end{array}\right)\left(\begin{array}{c}x_{1} \\ \vdots \\ x_{n}\end{array}\right)=T \boldsymbol{x}$. Then $Q\left(v_{0}\right)=\sum d_{i} y_{i}^{2} \leq d_{1}$ since $d_{1}=Q\left(e_{1}\right)$ is a rational number. This implies $\left|y_{i}\right| \leq \sqrt{d_{1} / d_{i}}$ and so $\boldsymbol{x}=$ $T^{-1} \boldsymbol{y}$ implies $\left|x_{i}\right|<c$, where $c$ is explicitely calculated. Since $B\left(e_{i}, e_{j}\right) \in$ $\boldsymbol{Q}$, taking a conjugate of the equation $Q\left(v_{0}\right)=\left(B\left(e_{i}, e_{j}\right)\right)[\boldsymbol{x}]$, we see $\left|x_{i}^{(j)}\right|$ $<c$, where $x_{i}^{(j)}$ is a conjugate of $x_{i}$. Denote by $S$ the set of algebraic integers $x$ with $1<[\boldsymbol{Q}(x): \boldsymbol{Q}] \leq m$ such that the absolute values of conjugates of $x$ is smaller than $c$. Then $S$ is a finite set. If $E$ contains no element of $S, x_{i}$ is rational. Hence $v_{0}$ is in $L$.

For simplicity we call $v_{0}$ in Lemma an element which gives the rational minimum of $O_{E} L$.

1.2. Proof of Theorem 1 . We may suppose that $L, M$ are positive definite by scaling if necessary. We assume that $E$ contains no element of $S$. We take an element $v_{0}$ of $O_{E} L$ which gives the rational minimum of $O_{E} L$. Let $\sigma$ be an isometry from $O_{E} L$ onto $O_{E} M ; \sigma\left(v_{0}\right)$ is an element which gives the rational minimum of $O_{E} M$. Let $O_{E}=Z\left[\omega_{1}, \cdots, \omega_{m}\right]$ and put $v_{0}=\sum \omega_{i} v_{i}$ and $L_{0}=Z\left[v_{1}, \cdots, v_{m}\right]$; the rank $L_{0} \leq m$ and $v_{0} \in O_{E} L_{0}$ is an element which gives the rational minimum of $O_{E} L_{0}$. Hence Lemma in 1.1 implies $v_{0} \in L_{0} \subset L$. Similarly we get $\sigma\left(v_{0}\right) \in M$. Now we have 
$\sigma\left(O_{E}\left(v_{0}^{\perp}\right.\right.$ in $\left.\left.L\right)\right)=\sigma\left(v_{0}^{\perp}\right.$ in $\left.O_{E} L\right)=\sigma\left(v_{0}\right)^{\perp}$ in $O_{E} M=O_{E}\left(\sigma\left(v_{0}\right)^{\perp}\right.$ in $\left.M\right)$. Theorem 1 is inductively proved.

2.1. The following lemma (Theorem 2.1 on p. 47 in [4]) is fundamental to prove Theorems 2,3 .

LEMMA. Let a be a totally real algebraic integer such that the absolute values of the conjugates over $\boldsymbol{Q}$ are less than 2 . Then $a$ is of the form $2 \cos r \pi(r \in \boldsymbol{Q})$.

Proof. Put $b=\sqrt{a^{2} / 4-1}$; then the assumption implies that $b^{2}$ is totally negative. Since $a / 2+b$ satisfies the equation $x^{2}-a x+1=0$, $a / 2+b$ is an algebraic integer and the absolute values of the conjugates are 1. Hence $a / 2+b$ is a root of the unity. This completes the proof.

COROLlARY. Let a be a totally real algebraic integer such that the absolute values of the conjugates are less than $\frac{16}{9}$. Then $a=0, \pm 1$, $\pm \sqrt{2}, \pm(1 \pm \sqrt{5}) / 2$ or $\pm \sqrt{3}$.

Proof. Lemma implies Corollary immediately.

2.2. Let $E$ be a totally real algebraic number field and $L$ be a positive definite quadratic lattice over $Z$ with $\operatorname{rank} L \leq 5$.

LEMMA. If $v_{0}$ is an element of $O_{E} L$ which gives the rational minimum of $O_{E} L$, then $v_{0}$ is in $L$ if $E$ does not contain any of $\sqrt{2}, \sqrt{3}$ and $\sqrt{5}$ in case of $\operatorname{rank} L=5$.

Proof. Put $n=\operatorname{rank} L$; then there is a basis $\left\{u_{i}\right\}$ of $L$ such that $\left(B\left(u_{i}, u_{j}\right)\right)=D[T]$, where $D=\left(\begin{array}{ll}d_{1} & \\ & \ddots \\ & \\ & d_{n}\end{array}\right)$ with $d_{i} / d_{i+1} \leq \frac{4}{3}(1 \leq i \leq n-1)$, $T=\left(\begin{array}{ccc}1 & & t_{i j} \\ & \ddots & \\ 0 & & 1\end{array}\right)\left(\left|t_{i j}\right| \leq \frac{1}{2}\right) . \quad$ Put $v_{0}=\sum \omega_{i} u_{i}, x=\left(\begin{array}{c}x_{1} \\ \vdots \\ x_{n}\end{array}\right)=T\left(\begin{array}{c}\omega_{1} \\ \vdots \\ \omega_{n}\end{array}\right) ;$ then $\sum d_{i} x_{i}^{2}$ $=Q\left(v_{0}\right) \leq d_{1}=Q\left(u_{1}\right)$. Since $Q\left(v_{0}\right)$ and $B\left(u_{i}, u_{j}\right)$ are rational, we have $\sum d_{i} x_{i}^{\prime 2}=Q\left(v_{0}\right) \leq d_{1}$, where $\left(\begin{array}{c}x_{1}^{\prime} \\ \vdots \\ x_{n}^{\prime}\end{array}\right)=T\left(\begin{array}{c}\omega_{1}^{\prime} \\ \vdots \\ \omega_{n}\end{array}\right)$ and $\left\{\omega_{i}^{\prime}\right\}$ is any conjugate of $\left\{\omega_{i}\right\}$. Let $n=2$; then we get $d_{2} x_{2}^{2} \leq d_{1}$. Hence $\left|\omega_{2}\right|=\left|x_{2}\right| \leq \sqrt{d_{1} / d_{2}} \leq$ $2 / \sqrt{3}$. Corollary in 2.1 implies $\omega_{2}=0$ or \pm 1 . If $\omega_{2}=0$, then $\omega_{1}=x_{1}$ 
and $d_{1} \omega_{1}^{2} \leq d_{1}$. Therefore $\omega_{1}=0$ or \pm 1 , and $v_{0} \in L$. If $\omega_{2}= \pm 1$, then $d_{1} x_{1}^{2} \leq d_{1}-d_{2}$. This yields $\left|x_{1}\right| \leq \sqrt{1-d_{2} / d_{1}} \leq \frac{1}{2}$, and $\left|\omega_{1}\right|=\left|x_{1}-t_{12} x_{2}\right| \leq$ $\frac{1}{2}+\frac{1}{2}=1$. Hence $\omega_{1}=0$ or \pm 1 and $v_{0}$ is in $L$. Let $n=3$; then $d_{3} x_{3}^{2}$ $\leq d_{1}$ implies $\left|\omega_{3}\right|=\left|x_{3}\right| \leq \sqrt{d_{1} / d_{3}} \leq \frac{4}{3}$. Hence $\omega_{3}=0$ or \pm 1 . If $\omega_{3}=0$, then $v_{0}$ is a vector which gives the rational minimum of $O_{E}\left[u_{1}, u_{2}\right]$, and so $v_{0}$ is in $Z\left[u_{1}, u_{2}\right] \subset L$. Suppose $\omega_{3}= \pm 1$; then $d_{2} x_{2}^{2} \leq d_{1}-d_{3}$ implies $\left|x_{2}\right| \leq \sqrt{d_{1} / d_{2}-d_{3} / d_{2}} \leq \sqrt{\frac{4}{3}-\frac{3}{4}}=\sqrt{\frac{7}{12}}$. Since $\omega_{2}=x_{2}-t_{23} \omega_{3},\left|\omega_{2}\right| \leq \sqrt{\frac{7}{12}}+$ $\frac{1}{2}$. Hence we get $\omega_{2}=0$ or \pm 1 , and $v_{0}$ is a vector which gives the rational minimum of $O_{E}\left[u_{1}, \omega_{2} u_{2}+\omega_{3} u_{3}\right]$. Hence $v_{0} \in Z\left[u_{1}, \omega_{2} u_{2}+\omega_{3} u_{3}\right] \subset L$. Let $n=4$; then $\left|\omega_{4}\right| \leq \sqrt{d_{1} / d_{4}} \leq \sqrt{\left(\frac{4}{3}\right)^{3}}$. Hence $\omega_{4}=0, \pm 1$ or $\pm \sqrt{2}$. As above we may assume $\omega_{4} \neq 0$. Since $\left|x_{3}\right| \leq \sqrt{d_{1} / d_{3}-d_{4} \omega_{4}^{2} / d_{3}} \leq \sqrt{\frac{16}{9}-\frac{3}{4} \omega_{4}^{2}}$, we have $\left|\omega_{3}\right| \leq \sqrt{\frac{16}{9}-\frac{3}{4} \omega_{4}^{2}}+\frac{1}{2}\left|\omega_{4}\right|$. Hence $\omega_{4}= \pm 1$ implies $\omega_{3}=0, \pm 1$ or $\pm \sqrt{2}$, and $\omega_{4}= \pm \sqrt{2}$ implies $\omega_{3}=0$ or \pm 1 . As above we may exclude the cases $\omega_{3}=0$, and $\left|\omega_{3}\right|=\left|\omega_{4}\right|=1$. Therefore we assume either $\left|\omega_{3}\right|=$ $\sqrt{2},\left|\omega_{4}\right|=1$ or $\left|\omega_{3}\right|=1,\left|\omega_{4}\right|=\sqrt{2}$. Since $x_{2}^{2} \leq d_{1} / d_{2}-d_{3} x_{3}^{2} / d_{2}-d_{4} x_{4}^{2} / d_{2}$ $\leq \frac{4}{3}-\frac{3}{4}\left(\omega_{3}+t_{34} \omega_{4}\right)^{2}-\frac{9}{16} \omega_{4}^{2}$, we get $x_{2}^{2} \leq-\frac{11}{12}+\frac{3}{4} \sqrt{2}$. Hence $\left|\omega_{2}\right|=\mid x_{2}-$ $t_{23} \omega_{3}-t_{24} \omega_{4} \mid \leq \sqrt{\frac{8}{4} \sqrt{2}-\frac{11}{12}}+\frac{1}{2}(1+\sqrt{2}) \leq 1.59$. This implies $\omega_{2}=0, \pm 1$ or $\pm \sqrt{2}$. If $\omega_{2}=0$ or \pm 1 , then $v_{0}$ is a vector which gives the rational minimum of $O_{E}\left[u_{1}, \omega_{2} u_{2}+\omega_{3} u_{3}, u_{4}\right]\left(\left|\omega_{4}\right|=\sqrt{2}\right)$ or $O_{E}\left[u_{1}, \omega_{2} u_{2}+\omega_{4} u_{4}, u_{3}\right]\left(\left|\omega_{4}\right|\right.$ $=1$ ), and so $v_{0}$ is in $L$. If $\left|\omega_{2}\right|=\sqrt{2}$, then $v_{0}$ is in $O_{E}\left[u_{1}, u_{3}, 1 / \sqrt{2}\left(\omega_{2} u_{2}\right.\right.$ $\left.\left.+\omega_{4} u_{4}\right)\right]\left(\left|\omega_{4}\right|=\sqrt{2}\right)$ or $O_{E}\left[u_{1}, u_{4}, 1 / \sqrt{2}\left(\omega_{2} u_{2}+\omega_{3} u_{3}\right)\right]\left(\left|\omega_{4}\right|=1\right)$, and $v_{0}$ is in $L$. Let $n=5$; then $d_{5} x_{5}^{2} \leq d_{1}$ implies $\left|\omega_{5}\right|=\left|x_{5}\right| \leq \frac{16}{9}$. From the assumption follows $\omega_{5}=0$ or \pm 1 . We may exclude $\omega_{5}=0$. Hence $x_{4}^{2} \leq d_{1} / d_{4}$ $-d_{5} / d_{4} \leq\left(\frac{4}{3}\right)^{3}-\frac{3}{4}$ and $\left|\omega_{4}\right| \leq\left|x_{4}-t_{45} \omega_{5}\right| \leq \sqrt{\left(\frac{4}{3}\right)^{3}-\frac{3}{4}}+\frac{1}{2} \leq \frac{16}{9}$, and so $\omega_{4}=0$ or \pm 1 . This yields $v_{0} \in L$. Thus, we have completed the proof.

2.3. Proof of Theorem 2. Without the loss of generality we may assume that $L, M$ are positive definite. If $v_{0}$ is an element which gives the rational minimum of $O_{E} L$, then $\sigma\left(v_{0}\right)$ is an element which gives the rational minimum of $O_{E} M$, where $\sigma$ is an isometry from $O_{E} L$ onto $O_{E} M$, so that Lemma in 2.2 implies $v_{0} \in L, \sigma\left(v_{0}\right) \in M$. By induction with respect to $\operatorname{rank} L=\operatorname{rank} M$ as in the proof of Theorem 1, we complete the proof of Theorem 2 .

2.4. Proof of Theorem 3. Let $E$ be a totally real algebraic number field with $[E: Q] \leq 5$, and $L, M$ be definite quadratic lattices over $Z$. As in 2.3 we may assume that $L, M$ are positive definite. To prove Theorem 
3 it suffices to show that an element which gives the rational minimum of $O_{E} L, O_{E} M$ is in $L, M$ respectively. Let $N=L$ or $M$ and $v_{0}$ be an element which gives the rational minimum of $O_{E} N$. Take a $Z$-basis $\left\{\omega_{1}, \cdots, \omega_{n}\right\}$ of $O_{E}$ and put $v_{0}=\sum \omega_{i} v_{i}$, where $v_{i} \in N$. Since $v_{0}$ is an element which gives the rational minimum of $O_{E} N_{0}, N_{0}=Z\left[v_{1}, \cdots, v_{n}\right]$, and rank $N_{0} \leq 5$, Lemma in 2.2 implies $v_{0} \in N_{0} \subset N$.

2.5. Remark. If Lemma in 2.2 is true without the restriction on the rank of $L$, our assumption (*) implies $\sigma(L)=M$ under the situation that $E$ is totally real, $F$ is the field $\boldsymbol{Q}$ of rational numbers, and $L, M$ are positive definite quadratic lattices over $Z$. The author knows no counterexamples.

2.6. Proof of Corollary of Theorem 2. Let $A$ be an ideal of $K$ such that $A$ is principal in the composite field $K E$. Since discriminants of $K, E$ are relatively prime, $O_{K E}=O_{K} O_{E}$. Hence we get $O_{K E} A=O_{E} A$ $=\lambda O_{E} O_{K}$, where $\lambda$ is an element of $K E$. Putting $A=Z\left[u_{1}, u_{2}\right], O_{K}=$ $Z\left[v_{1}, v_{2}\right]$, we have $\left(\lambda v_{1}, \lambda v_{2}\right)=\left(u_{1}, u_{2}\right)\left(\begin{array}{ll}a & b \\ c & d\end{array}\right)$, where $\left(\begin{array}{ll}a & b \\ c & d\end{array}\right) \in G L\left(2, O_{E}\right)$. Let $x, y \in O_{E}$ and $N A$ be the norm of $A$ in $K$; then

$$
\begin{aligned}
& N A^{-1}\left|(x a+y b) u_{1}+(x c+y d) u_{2}\right|^{2} \\
& \quad=N A^{-1}\left|\lambda\left(v_{1} x+v_{2} y\right)\right|^{2}=|\lambda|^{2} N A^{-1}\left|v_{1} x+v_{2} y\right|^{2} .
\end{aligned}
$$

On the other hand, the discriminants of binary quadratic forms $N A^{-1} \mid x u_{1}$ $+\left.y u_{2}\right|^{2},\left|v_{1} x+v_{2} y\right|^{2}$ are equal to the discriminant of $K$. Comparing the both sides of the above equation, we have $(a d-b c)^{2}=\left(|\lambda|^{2} N A^{-1}\right)^{2}$. Put $\left(\begin{array}{ll}a^{\prime} & b^{\prime} \\ c^{\prime} & d^{\prime}\end{array}\right)=\sqrt{|\lambda|^{-2} N A}\left(\begin{array}{ll}a & b \\ c & d\end{array}\right)$; then $a^{\prime} d^{\prime}-b^{\prime} c^{\prime}= \pm 1$ and $N A^{-1} \mid\left(x a^{\prime}+y b^{\prime}\right) u_{1}$ $+\left.\left(x c^{\prime}+y d^{\prime}\right) u_{2}\right|^{2}=\left|v_{1} x+v_{2} y\right|^{2}$. Moreover $|\lambda|^{2}$ is totally positive since $|\lambda|^{2}$ $=\alpha^{2}+\delta \beta^{2}$ where $\lambda=\alpha+\sqrt{-\delta} \beta, K=\boldsymbol{Q}(\sqrt{-\delta})$. In other words, the binary positive definite quadratic forms associated with the ideal $A, O_{K}$ are equivalent over $O_{E\left(\sqrt{|\lambda|^{2} A^{-1}}\right)}$. From Theorem 2 follows that they are equivalent over $Z$. This means that $A$ is principal.

3. Proof of Theorem 5. Without the loss of generality we may assume that $L, M$ be positive definite at each infinite place by scaling.

Suppose that $O_{E}$ satisfies the first condition (i). Regarding $O_{E} L$ as a quadratic lattice over $O_{F}$ with bilinear form $\operatorname{tr}_{E / F} B(x, y), O_{E} L$ has the orthogonal decomposition to indecomposable quadratic lattices $O_{E} L=L_{1}$ 
$\perp \cdots \perp L_{m} \perp A \sqrt{a} L_{1} \perp \cdots \perp A \sqrt{a} L_{m}$, where $L=L_{1} \perp \cdots \perp L_{m}$ is the orthogonal decomposition of $L$ to indecomposable quadratic lattices. Let $M=M_{1} \perp \cdots \perp M_{n}$ be the orthogonal decomposition of $M$ to indecomposable quadratic lattices. The isometry $\sigma$ gives an isometry from $O_{E} L$ onto $O_{E} M$, which are regarded as definite quadratic lattices over $O_{F}$ as above, and so $n=m$. If $\sigma L_{1}=M_{i}$, then $\sigma\left(O_{E}\left(\perp_{i z_{2}} L_{i}\right)\right)=O_{E}\left(\perp_{j \neq i} M_{j}\right)$, and Lemma is inductively proved. Hence we may assume $\sigma\left(L_{i}\right)=$ $A \sqrt{a} M_{\alpha(i)}$, where $\alpha(i)$ is a permutation of the set $\{1,2, \cdots, m\}$. Thus we have $\sigma(L)=A \sqrt{a} M$. Comparing the volumes of the both sides, $(A \sqrt{a})^{2}=O_{F}$. This is a contradiction. Next we suppose the second condition (ii) on $O_{E}$. Put $\sigma(v)=\varphi_{1}(v)+x \varphi_{2}(v)$, where $v \in L, \varphi_{1}(v) \in M$, $\varphi_{2}(v) \in A M$. We may assume $x=\sqrt{a}+b(0 \neq b \in F)$. Since $Q(v)=$ $Q(\sigma(v))=Q\left(\varphi_{1}(v)\right)+2 x B\left(\varphi_{1}(v), \varphi_{2}(v)\right)+x^{2} Q\left(\varphi_{2}(v)\right) \in F$, we get $Q(v)=Q\left(\varphi_{1}(v)\right)$ $+2 b B\left(\varphi_{1}(v), \varphi_{2}(v)\right)+\left(a+b^{2}\right) Q\left(\varphi_{2}(v)\right), B\left(\varphi_{1}(v), \varphi_{2}(v)\right)+b Q\left(\varphi_{2}(v)\right)=0$. Thus $Q(v)=Q\left(\varphi_{1}(v)\right)+\left(a-b^{2}\right) Q\left(\varphi_{2}(v)\right)=Q\left(\varphi_{1}(v)\right)-N_{E / F}(x) Q\left(\varphi_{2}(v)\right)$, and we see that $\varphi_{1}$ is injective. From our assumption follows that $Q(v)-Q\left(\varphi_{1}(v)\right)$ is zero or totally positive. Similarly we get an injective mapping $\varphi_{1}^{\prime}$ from $M$ to $L$ such that $Q(v)-Q\left(\varphi_{1}^{\prime}(v)\right)$ is zero or totally positive if $v \in M$. Put $\varphi=\varphi_{1}^{\prime} \varphi_{1}$; then $\varphi$ is an injective endomorphism of $L$ such that $Q(v)$ $-Q(\varphi(v))$ is zero or totally positive. Let $\left\{v_{i}\right\}$ be elements of $L$ such that $\left[L: O_{F}\left[\cdots, v_{i}, \cdots\right]\right]<\infty$. From the property of $\varphi$ follows that there is a natural number $k$ such that $Q\left(\varphi^{k}\left(v_{i}\right)\right)=Q\left(\varphi^{k+1}\left(v_{i}\right)\right)$ for any index $i$. Since $\varphi$ is monomorphism, we may suppose that $v_{i}$ themselves satisfy $\left[L ; O_{F}\left[\cdots, v_{i}, \cdots\right]\right]<\infty$ and $Q\left(v_{i}\right)=Q\left(\varphi\left(v_{i}\right)\right)$ instead of $\varphi^{k}\left(v_{i}\right)$. Thus

$$
Q\left(v_{i}\right)-Q\left(\varphi\left(v_{i}\right)\right)=Q\left(v_{i}\right)-Q\left(\varphi_{1}\left(v_{i}\right)\right)+Q\left(\varphi_{1}\left(v_{i}\right)\right)-Q\left(\varphi_{1}^{\prime} \varphi_{1}\left(v_{i}\right)\right)=0
$$

implies $Q\left(v_{i}\right)=Q\left(\varphi_{1}\left(v_{i}\right)\right)$ and $Q\left(\varphi_{2}\left(v_{i}\right)\right)=0$. Hence we get $\varphi_{2}\left(v_{i}\right)=0$ and $\varphi_{2}=0$. This means $\sigma(L) \subset M$. Similarly we get $\sigma^{-1}(M) \subset L$. Hence $\sigma(L)=M$.

4. Proof of Theorem 4. Let $E / F$ be a Galois extension of totally real algebraic number fields satisfying the assumption in Theorem 4 , and $L$ be an indecomposable definite quadratic lattice over $O_{F}$. It suffices to prove $O_{E} L$ is still indecomposable. Suppose that $O_{E} L$ is not indecomposable, i.e., $O_{E} L=L_{1} \perp \cdots \perp L_{m}(m>1)$, where each $L_{i}$ is indecomposable. Denote by $G$ the Galois group $G(E / F)$ and operate $G$ on $O_{E} L$ as follows: $g(a v)=g(a) v$ for $a \in O_{E}, v \in L, g \in G$. Then $g B(x, y)=B(g(x)$, $g(y))\left(x, y \in O_{E} L\right)$ implies $O_{E} L=g\left(L_{1}\right) \perp \cdots \perp g\left(L_{m}\right)$ for $g \in G$. From the 
uniqueness of the orthogonal decomposition of a definite quadratic lattice to indecomposable lattices follows $g\left(L_{i}\right)=L_{g(i)}$, where $g(i)$ stands for a permutation. If $G$ does not operate on the set $\left\{L_{i}\right\}$ transitively, then there is a decomposition $O_{E} L=L_{1}^{\prime} \perp L_{2}^{\prime}$, where $L_{i}^{\prime}$ is $G$-invariant as a set. Let $v$ be an element of $L$; then $v=v_{1}+v_{2}\left(v_{1} \in L_{1}^{\prime}, v_{2} \in L_{2}^{\prime}\right)$. Since $v=g v=g\left(v_{1}\right)+g\left(v_{2}\right)$ and $g\left(v_{i}\right) \in L_{i}^{\prime}$ for any $g$ in $G, g v_{i}=v_{i}$ for any $g$ in $G$. This implies $v_{i} \in L$. Hence $L=\left(L_{1}^{\prime} \cap L\right) \perp\left(L_{2}^{\prime} \cap L\right)$. This contradicts our assumption. Thus $G$ operates on the set $\left\{L_{i}\right\}$ transitively. Put $H=\left\{g \in G ; g L_{1}=L_{1}\right\}$ and $g_{i} L_{1}=L_{i}, G=\bigcup_{i=1}^{m} g_{i} H$. From our assumption $m>1$ follows $H \neq G$. Put $K=\{a \in E ; h(a)=a$ for any $h$ in $H\}$, and $M_{1}=\left\{v \in L_{1} ; h(v)=v\right.$ for any $h$ in $\left.H\right\}$. Let $v=\tilde{v}_{1}+\tilde{v}_{2}$ be an element in $O_{K} L\left(\tilde{v}_{1} \in L_{1}, \tilde{v}_{2} \in \perp_{i \geq 2} L_{i}\right)$; then $v=h(v)=h\left(\tilde{v}_{1}\right)+h\left(\tilde{v}_{2}\right)$ implies $h\left(\tilde{v}_{i}\right)=\tilde{v}_{i}(i=1,2)$ for any $h$ in $H$. Hence $O_{K} L=M_{1} \perp\left(O_{K} L \cap \perp_{i \geq 2} L_{i}\right)$. This implies $L_{1}=O_{E} M_{1}$. Let $M_{1}=O_{K} v_{1} \oplus \cdots \oplus O_{K} v_{r-1} \oplus A v_{r}$, where $A$ is an ideal of $K$ such that $A$ and the relative discriminant $D(E / F)$ of $E / F$ are relatively prime. Similarly let $L=O_{F} u_{1} \oplus \cdots \oplus O_{F} u_{n-1} \oplus B u_{n}$ $(n=m r)$, where $B$ is an ideal of $F$ such that $B$ and $D(E / F)$ are relatively prime. Put $v_{i}=\sum_{j=1}^{n} a_{i j} u_{j}\left(a_{i j} \in E\right)$, and $u_{i}=\sum_{j=1}^{m} g_{j}\left(w_{j, i}\right)$, where $w_{j, i}$ is an element of $L_{1}$. Since $v_{i}$ is fixed by $H$ and $g u_{i}=u_{i}(g \in G), a_{i j}$ is an element of $K$, and $u_{i}=\sum_{j} g_{j}\left(w_{j, i}\right)=\sum_{j} g g_{j}\left(w_{j, i}\right)(g \in G)$. Comparing the components in $L_{1}$, we get $w_{1, i}=h\left(w_{j, i}\right)(h \in H)$. Thus $u_{j}$ is written as $\sum_{k=1}^{m} g_{k}\left(w_{j}\right)\left(w_{j} \in M_{1}\right)$. Putting $w_{j}=\sum_{t=1}^{r} b_{j t} v_{t}\left(b_{j t} \in K\right)$, we have $v_{i}=\sum_{j=1}^{n} a_{i j} u_{j}=\sum_{j=1}^{n} \sum_{k=1}^{m} a_{i j} g_{k}\left(w_{j}\right)=\sum_{j=1}^{n} \sum_{k=1}^{m} \sum_{t=1}^{r} a_{i j} g_{k}\left(b_{j t}\right) g_{k}\left(v_{t}\right)$. Thus $v_{i}=\sum_{j=1}^{n} \sum_{t=1}^{r} a_{i j} b_{j t} v_{t}, \sum_{j=1}^{n} \sum_{t=1}^{r} a_{i j} g_{k}\left(b_{j t}\right) g_{k}\left(v_{t}\right)=0$ if $k>1$. If we put $a_{j}=a_{1, j}, b_{j}=b_{j, 1}$, then $\sum_{j=1}^{n} a_{j} b_{j}=1$ and $\sum_{j=1}^{n} g\left(a_{j}\right) b_{j}=0$ if $g \notin H$. From our assumption on ideals $A, B$ follows that $a_{i}, b_{i}$ are $\mathfrak{p}$-adic integers of $K$ if $\mathfrak{p} \mid D(E / F)$. Let $O_{K}=O_{F} \omega_{1} \oplus \cdots \oplus O_{F} \omega_{m-1} \oplus C \omega_{m}$, where $C$ is an ideal of $F$ such that $C$ and $D(E / F)$ are relatively prime, and $a_{i}=$ $\sum_{j=1}^{m} c_{i j} \omega_{j}, \quad b_{i}=\sum_{t=1}^{m} d_{i t} \omega_{t}$ and $f_{j t}=\sum_{i=1}^{n} c_{i j} d_{i t} ;$ then $\sum_{j, t=1}^{m} f_{j t} \omega_{j} \omega_{t}=1$, $\sum_{j, t=1}^{m} f_{j t} g\left(\omega_{j}\right) \omega_{t}=0(g \notin H)$, and $f_{i j}$ is a $p$-adic integer of $F$ if $\mathfrak{p} \mid D(E / F)$. Putting $\Omega=\left(\omega_{1}, \cdots, \omega_{m}\right),{ }^{t} \Omega^{\prime}=\left(\sum_{t=1}^{m} f_{1 t} \omega_{t}, \cdots, \sum_{t=1}^{m} f_{m t} \omega_{t}\right)$, we have $\Omega \Omega^{\prime}$ $=1, g(\Omega) \Omega^{\prime}=0$ if $g \notin H$. Hence we get

$$
\left(\begin{array}{c}
g_{1}(\Omega) \\
\vdots \\
g_{m}(\Omega)
\end{array}\right) \Omega^{\prime}=\left(\begin{array}{c}
1 \\
0 \\
\vdots \\
0
\end{array}\right)=e .
$$

We define a permutation matrix $M_{i}$ by 


$$
\left(\begin{array}{c}
g_{i} g_{1}(\Omega) \\
\vdots \\
g_{i} g_{m}(\Omega)
\end{array}\right)=M_{i}\left(\begin{array}{c}
g_{1}(\Omega) \\
\vdots \\
g_{m}(\Omega)
\end{array}\right)
$$

Then $M_{i}\left(\begin{array}{c}g_{1}(\Omega) \\ \vdots \\ g_{m}(\Omega)\end{array}\right) g_{i}\left(\Omega^{\prime}\right)=e$ implies

$$
\left(\begin{array}{c}
g_{1}(\Omega) \\
\vdots \\
g_{m}(\Omega)
\end{array}\right)\left(g_{1}\left(\Omega^{\prime}\right), \cdots, g_{m}\left(\Omega^{\prime}\right)\right)=\left(M_{1}^{-1} e, \cdots, M_{m}^{-1} \boldsymbol{e}\right)
$$

If $g\left(\Omega^{\prime}\right)=\Omega^{\prime}$, then $g \in H$ since $\Omega \Omega^{\prime}=1$ and $g(\Omega) \Omega^{\prime}=1$. If $M_{i}^{-1} e=M_{j}^{-1} e$, then $\left(\begin{array}{c}g_{1}(\Omega) \\ \vdots \\ g_{m}(\Omega)\end{array}\right) g_{i}\left(\Omega^{\prime}\right)=\left(\begin{array}{c}g_{1}(\Omega) \\ \vdots \\ g_{m}(\Omega)\end{array}\right) g_{j}\left(\Omega^{\prime}\right)$ and $g_{i}\left(\Omega^{\prime}\right)=g_{j}\left(\Omega^{\prime}\right)$. Therefore $i=j$, and this means that $\left(M_{1}^{-1} \boldsymbol{e}, \cdots, M_{m}^{-1} \boldsymbol{e}\right)$ is a permutation matrix. Thus we have

$$
\operatorname{det}\left(\begin{array}{c}
g_{1}(\Omega) \\
\vdots \\
g_{m}(\Omega)
\end{array}\right) \cdot \operatorname{det}\left(g_{1}\left(\Omega^{\prime}\right), \cdots, g_{m}\left(\Omega^{\prime}\right)\right)= \pm 1 .
$$

From our assumption follows that both components on the left side are $\mathfrak{p}$-adic integers if $\mathfrak{p} \mid D(E / F)$. Hence $\operatorname{det}\left(\begin{array}{c}g_{1}(\Omega) \\ \vdots \\ g_{m}(\Omega)\end{array}\right)$ is a $p$-adic unit if $\mathfrak{p} \mid D(E / F)$, and this implies that $K$ is unramified over $F$. This is a contradiction.

4.1. Proof of Corollary of Theorem 4. If $E$ is a totally real algebraic number field, then there is a totally real algebraic number field $\tilde{E}$ which is a Galois extension of $\boldsymbol{Q}$ and contains $E$. If $O_{E} L$ is decomposable, then $O_{\tilde{E}} L$ is also decomposable. This contradicts Theorem 4.

4.2. If $E / F$ is an unramified extension of totally real algebraic number fields, then we will show that there is an indecomposable definite quadratic lattice over $O_{F}$ which is decomposable over $O_{E}$. We take an unramified Galois extension $K / F$ where $K$ is totally real and contains $E$. Denote by $G=\left\{g_{1}=1, g_{2}, \cdots, g_{m}\right\}$ the Galois group of $K / F$. Let $V$ be 
an $m$-dimensional quadratic space over $F$ with orthonormal basis $\left\{v_{i}\right\}$. We define the operation of $G$ to $K V$ by $g_{i}\left(a v_{1}\right)=g_{i}(a) v_{i}(a \in K)$. Put $\tilde{L}=O_{K} v_{1} \perp \cdots \perp O_{K} v_{m}(\subset K V)$ and $L=\left\{\sum_{i=1}^{m} g_{i}(a) v_{i} ; a \in O_{K}\right\}$. By definition, $G$ operates trivially on $L$. Since $K / F$ is unramified, there are elements $a_{1}, \cdots, a_{m}$ in $O_{K}$ such that $\left(g_{i}\left(a_{j}\right)\right)$ is a unimodular matrix at a given prime. Hence $\tilde{L}=O_{K} L$. If $L$ is decomposable, and $L=L_{1} \perp L_{2}$, then $O_{K} L_{1}$ is an orthogonal sum of a proper subset of $\left\{O_{K} v_{i}\right\}$. Thus $O_{K} L_{1}$ is not closed under the operation of $G$. This is a contradiction because $G$ operates trivially on $L_{1}$. Denote the subgroup of $G$ corresponding to $E$ by $H$. Let $G=\bigcup H h_{i}$ (coset decomposition) and put $\tilde{L}_{i}$ $=\perp_{h \in H} O_{K} h h_{i}\left(v_{1}\right)$ which is closed under the operation of $H$; then $\tilde{L}=$ $\perp_{i} \tilde{L}_{i}$. Decompose an element $u$ in $O_{E} L$ as $u=\sum u_{i}, u_{i} \in \tilde{L}_{i}$. By definition $u=h(u)=\sum h\left(u_{i}\right)$ for $h$ in $H$. Hence we get $h\left(u_{i}\right)=u_{i}$ and $O_{E} L$ $=\perp\left(\tilde{L}_{i} \cap O_{E} L\right)$. Thus $O_{E} L$ is decomposable.

5. Proof of Theorem 6. Put $N=L \perp M$ and $\tilde{\sigma}$ be an isometry of $O_{E} N$ defined as $\tilde{\sigma}(u+m)=\sigma(u)+\sigma^{-1}(m)$ for $u \in O_{E} L, m \in O_{E} M$. If $\tilde{\sigma}(v)$ $\equiv v \bmod 2 O_{E} N$ for $v \in O_{E} N$, then $v=(v+\tilde{\sigma}(v)) / 2+(v-\tilde{\sigma}(v)) / 2 \in N_{+} \perp$ $N_{-}$, where $N_{ \pm}=\left\{u \in O_{E} N ; \tilde{\sigma}(u)= \pm u\right\}$. Thus $N_{+} \perp N_{-}=\left\{v \in O_{E} N ; \tilde{\sigma}(v)\right.$ $\left.\equiv v \bmod 2 O_{E} N\right\}$. Since $v=u_{1}+\sigma\left(u_{2}\right)\left(u_{1}, u_{2} \in O_{E} L\right)$ is in $N_{+} \perp N_{-}$if and only if $u_{1} \equiv u_{2} \bmod 2 O_{E} L$, we get $N_{+} \perp N_{-}=O_{E}\{u+\sigma(u) ; u \in L\}+2 O_{E} M$. Our assumption implies $\sigma(u)=m_{u}+2 v$ for $u \in L$, where $m_{u} \in M, v \in O_{E} M$. Thus $N_{+} \perp N_{-}=O_{E}\left\{\left\{u+m_{u} ; u \in L\right\}+2 M\right\}$. By virtue of Theorem 4, there are sublattices $\tilde{N}_{+}, \tilde{N}_{-}$of $N$ such that $\left\{u+m_{u} ; u \in L\right\}+2 M=\tilde{N}_{+}$ $\perp \tilde{N}_{-}$and $N_{+}=O_{E} \tilde{N}_{+}, N_{-}=O_{E} \tilde{N}_{-}$. So, $\tilde{\sigma}= \pm 1$ on $N_{ \pm}$implies $\tilde{\sigma}\left(\tilde{N}_{+}\right)$ $=\tilde{N}_{+}, \tilde{\sigma}\left(\tilde{N}_{-}\right)=\tilde{N}_{-} . \quad$ Thus $\tilde{\sigma}(F L \perp F M)=F L \perp F M$. Therefore $\tilde{\sigma}(L \perp M)$ $=L \perp M$. This yields $\sigma L=M$.

6. Remark. Theorems 2, 3 are fairly improved by a different method which will appear in a subsequent paper.

\section{REFERENCES}

[1] A. Borel, Introduction aux groupes arithmétiques, Hermann, Paris, 1967.

[2] A. G. Earnest and J. S. Hsia, Springer-type theorems for spinor genera of quadratic forms, Bull. Amer. Math. Soc., 81 (1975), 942-943.

[ 3 ] A. G. Earnest and J. S. Hsia, Spinor genera under field extension (to appear in Acta Arith.).

[4] W. Narkiewicz, Elementary and analytic theory of algebraic numbers, Warszawa, 1974.

[5] O. T. O'Meara, Introduction to quadratic forms, Springer-Verlag, Berlin, 1963. 
[6] T. A. Springer, Sur les formes quadratiques d'indice zéro, C. R. Acad. Sci., 234 (1952), 1517-1519.

Department of Mathematics

Nagoya University 